\title{
A note on Krasnosel'skii fixed point theorem
}

Tian Xiang ${ }^{1 *}$ and Rong Yuan ${ }^{2}$

"Correspondence: txiang@ruc.edu.cn

${ }^{1}$ Institute for Mathematical Sciences, Renmin University of China, Beijing, 100872, China Full list of author information is available at the end of the article

\begin{abstract}
In this note, a couple of unclear and unnecessary points made in the two existing papers by Liu and Li (Proc. Am. Math. Soc. 136:1213-1220, 2008) and Xiang and Yuan (Proc. Am. Math. Soc. 139:1033-1044, 2011) are first pointed out and clarified. Second, a few additional remarks are observed. Upon these observations, three corresponding refined and unified Krasnosel'skii fixed point theorems in strong topology setup are formulated. As an illustration, several new classes of Krasnosel'skii fixed point theorems are obtained, which expand and complement some known related results by Agarwal, O'Regan and Taoudi (Fixed Point Theory Appl. 2010:243716, 2010) and Edmunds (Math. Ann. 174:233-239, 1967).
\end{abstract}

MSC: $47 \mathrm{H} 10 ; 47 \mathrm{H} 08 ; 47 \mathrm{HO}$

Keywords: Krasnosel'skii fixed point theorem; noncompact mapping; multi-valued mapping; nonexpansive

\section{Introduction}

Sixty years ago, Krasnosel'skii [1, 2] observed that many problems in analysis can be formulated abstractly as a mapping which is the sum of a contraction and a compact map. He proved a fixed point theorem covering such cases which combines the Banach contraction mapping principle and Schauder's fixed point theorem. Since then, more and more scholars are interested in the study of the existence of solutions of nonlinear abstract operator equation or the fixed point for the sum of two operators of the form

$$
S x+T x=x, \quad x \in K,
$$

where $K$ is a closed and convex subset of a Banach space $E$. The Krasnosel'skii fixed point theorem appears as a prototype for solving equations of the form (1.1) and it initiates vast investigations in the direction of such mixed type problems.

In the literature, there appears a huge number of papers studying the fixed point problems for the sum of two operators, the generalizations and its variants of Krasnosel'skii fixed point theorem and their applications in real-world problems, see, for instance, [3-12] and the references therein. In 2008, a quite general compact-type Krasnosel'skii fixed point theorem was obtained by Liu and Li in [6]. They imposed a compactness hypothesis on the operator $S$ and some other properties on the operator $I-T$, and then asserted the following result.

(c) 2015 Xiang and Yuan. This article is distributed under the terms of the Creative Commons Attribution 4.0 International License (http://creativecommons.org/licenses/by/4.0/), which permits unrestricted use, distribution, and reproduction in any medium, provided you give appropriate credit to the original author(s) and the source, provide a link to the Creative Commons license, and indicate if changes were made. 
Theorem 1.1 Let $K$ be a bounded, closed and convex nonempty subset of a Banach space $(E,\|\cdot\|)$. Suppose that $T$ and $S$ are continuous and map $K$ into $E$ such that

(a) $S(K) \subset(I-T)(K)$;

(b) $S(K)$ is contained in a compact subset of $K$;

(c) if $(I-T) x_{n} \rightarrow y$, then there exists a convergent subsequence $\left\{x_{n_{k}}\right\}$ of $\left\{x_{n}\right\}$;

(d) for every $y$ in the range of $(I-T), D_{y}=\{x \in K:(I-T) x=y\}$ is a convex set.

Then there exists a point $x^{*} \in K$ with $S x^{*}+T x^{*}=x^{*}$.

In 2011, a noncompact-type Krasnosel'skii fixed point theorem was established by the authors in [9]. There we first relaxed the compactness assumption of $S$ and derived a generalized noncompact-type Krasnosel'skii fixed point theorem.

Theorem 1.2 Let $K \subset E$ be a nonempty, bounded, closed and convex subset and $T: E \rightarrow E$ be a map. Suppose that $S: K \rightarrow E$ is continuous such that

(i) $S(K) \subset(I-T)(E)$ and $[x=T x+S y, y \in K] \Longrightarrow x \in K($ or $S(K) \subset(I-T)(K))$;

(ii) $\psi(T(A)+S(A))<\psi(A)$ for all $A \subset K$ with $\psi(A)>0$;

(iii) if $\left\{x_{n}\right\}$ is a sequence in $\mathcal{F}(E, K ; T, S)$ and $(I-T) x_{n} \rightarrow y$, then $\left\{x_{n_{k}}\right\}$ is convergent, where $\left\{x_{n_{k}}\right\}$ is a subsequence of $\left\{x_{n}\right\}$;

(iv) $T$ is closed in $\mathcal{F}$; that is, if $\left\{x_{n}\right\} \subset \mathcal{F}$ for which $x_{n} \rightarrow x$ and $T x_{n} \rightarrow y$, then $y=T x$;

(v) for each $y$ in the range of $S$, the set $\Delta_{y}=\{x \in E:(I-T) x=y\}$ is convex.

Then there exists a point $x^{*} \in K$ with $S x^{*}+T x^{*}=x^{*}$.

Upon scrutinizing the proof of Theorem 1.2, we then placed a suitable compactness on the possible fixed point set $\mathcal{F}(E, K ; T, S)$ and obtained an extension of the preceding theorem. In the above notations, $\psi$ denotes either the Kuratowskii or Hausdorff measure of non-compactness and $\mathcal{F}(E, K ; T, S)=\{x \in E: x=T x+S y$ for some $y \in K\}$.

First, a careful reading of the aforementioned papers reveals the following five items.

$\mathrm{p}_{1}$. Several unclear and unnecessary points made in [6] are pointed out and clarified. Specifically, when $I-T$ is invertible, they claimed that it is easy to see by item (c) that $(I-T)^{-1}$ is continuous. However, this assertion is a little vague since, on the one hand, it is not clear whether $\left\{x_{n}\right\}$ belongs to $S(K)$ or to $(I-T)(K)$; on the other hand, it is also not clear where $(I-T)^{-1}$ is continuous, see Remarks 2.1 and 2.2 below. Moreover, the assertion turns out to be not so said easy as we shall see later. In addition, the condition (b) is a little restrictive and it is clearly sufficient to require that $S(K)$ is contained in a compact subset of $E$ instead of $K$. The condition (d) is also somewhat demanding and it can be lessened by the following:

$\left(\mathrm{d}^{\prime}\right)$ for every $z$ in the range of $S, D_{z}=\{x \in K:(I-T) x=z\}$ is a convex set.

The assumption ( $\left.\mathrm{d}^{\prime}\right)$ suffices to ensure that the values of the possible multi-valued mapping $(I-T)^{-1} S: K \rightarrow P(K)$ (the power set of $K$ ) are convex. Finally, the requirement that $K$ be bounded is not necessary under the conditions of Theorem 1.1.

$\mathrm{p}_{2}$. Under the assumptions of Theorem 1.2, the seemingly important assumption (iii) is proved to be completely redundant, and therefore can be removed from the theorem without affecting its conclusion. So is the hypothesis (iii) of Theorem 2.3 in [9]. Also, $T$ can be defined on a superset $M$ of $K$, not necessarily on the whole space $E$. 
$\mathrm{p}_{3}$. Several correlations between the relative compactness of the set $\mathcal{F}(E, K ; T, S)$ and the relative compactness of the set $S(K)$ are observed, see Proposition 2.1 below.

$\mathrm{p}_{4}$. If we can establish a nonempty, compact, possible fixed point subset of the set $\mathcal{F}(E, K ; T, S)$ or even of $\mathcal{F}(M, K ; T, S)$ with $M \supset K$, then the above (iii) is not needed as indicated in the second point above. Otherwise, if a compactness assumption is placed only on the set $S(K)$, then the condition (iii) above becomes necessary.

$\mathrm{p}_{5}$. When the compactness condition is placed on the $\operatorname{set} \mathcal{F}(E, K ; T, S)$, it will be illustrated that $(I-T)^{-1}$ needs not be continuous even on $S(K)$; whereas if the compactness hypothesis is imposed on $S(K)$, then $(I-T)^{-1}$ may not be continuous on $(I-T)(K)$, see also Remark 2.2 below.

The item $\mathrm{p}_{1}$ is comparable with Remark 2.3 in [9] and it is a detailed account of it. Then, upon these refined formulations of the Krasnosel'skii fixed point theorems, we can easily derive new classes of Krasnosel'skii type theorems. Here, as an illustration, we present one paradigm of them, see Theorem 2.4; also, we provide different conditions to establish two fixed point results for $T+S$ with $T$ being nonexpansive, see Theorems 2.5 and 2.6, which extend and complement the corresponding results of $[7,13]$.

In the end of the note, three simple and interesting examples are offered to show that the continuity of the composite mapping $T \circ S$ does not necessarily ensure the continuity of its components $T$ and $S$. This makes the clarifications above necessary.

\section{Refined Krasnosel'skii fixed point theorems and their corollaries}

We first consider the case where the compactness condition is imposed on the operator $S$. The observations made in items $\mathrm{p}_{1}$ and $\mathrm{p}_{4}$ lead us to formulate the following proofread and generalized compact-type Krasnosel'skii fixed point theorem.

Theorem 2.1 Let $K \subset E$ be a nonempty, bounded, closed and convex subset and $K \subset$ $M \subset E$. Suppose that $T: M \rightarrow E$ and $S: K \rightarrow E$ are two mappings such that

(i) $S(K) \subset(I-T)(M)$ and $[x=T x+S y, y \in K] \Longrightarrow x \in K($ or $S(K) \subset(I-T)(K))$;

(ii) $S$ is continuous and $S(K)$ resides in a compact subset of $E$;

(iii) if $\left\{x_{n}\right\}$ is a sequence in $\mathcal{F}(M, K ; T, S)$ and $(I-T) x_{n} \rightarrow y$, then $\left\{x_{n}\right\}$ possesses a convergent subsequence $\left\{x_{n_{k}}\right\}$;

(iv) if $\left\{x_{n}\right\} \subset \mathcal{F}$ for which $x_{n} \rightarrow x$ and $T x_{n} \rightarrow y$, then $y=T x$;

(v) for each $z \in S(K)$, the set $\Delta_{z}=\{x \in M:(I-T) x=z\}$ is convex.

Then there exists a point $x^{*} \in K$ so that $S x^{*}+T x^{*}=x^{*}$.

Proof It suffices to show, when $I-T$ is one-to-one, that $(I-T)^{-1}: S(K) \rightarrow K$ is continuous. The fact that $(I-T)^{-1}$ transforms $S(K)$ into $K$ is justified by the condition (i). Let now $y_{n}, y \in S(K)$ with $y_{n} \rightarrow y$, and let $x_{n}=(I-T)^{-1} y_{n}$ and $x=(I-T)^{-1} y$. The definition of $\mathcal{F}$ implies that $x_{n} \in \mathcal{F}$ and $(I-T) x_{n} \rightarrow y$. The assumption (iii) then shows that $\left\{x_{n}\right\}$ has a subsequence $\left\{x_{n_{k}}\right\}$ converging to some $x_{0} \in K$. Accordingly $T x_{n_{k}} \rightarrow x_{0}-y$. The closedness of $T$ in $\mathcal{F}$ (cf. item (iv)) therefore gives us that $x_{0}-y=T x_{0}$, i.e., $x_{0}=(I-T)^{-1} y$. Evidently $x_{0}=x$ since $I-T$ is injective. Summing up the above arguments, we have derived

$$
(I-T) y_{n_{k}} \rightarrow(I-T)^{-1} y
$$


We next claim that

$$
(I-T) y_{n} \rightarrow(I-T)^{-1} y .
$$

Suppose the contrary, then there exists a neighborhood $U$ of $(I-T)^{-1} y$ and a subsequence $\left\{y_{n_{j}}\right\}$ of $\left\{y_{n}\right\}$ such that $(I-T)^{-1} y_{n_{j}} \notin U$ for all $j \geq 1$. Naturally, $\left\{y_{n_{j}}\right\}$ converges to $y$, then reasoning as before we may extract a subsequence $\left\{y_{n_{j_{k}}}\right\}$ of $\left\{y_{n_{j}}\right\}$ so that $(I-T)^{-1} y_{n_{j_{k}}} \rightarrow$ $(I-T)^{-1} y$. But this is a contradiction since $(I-T)^{-1} y_{n_{j}} \notin U$ for all $j \geq 1$. The claim is hence confirmed, and finally $(I-T)^{-1}: S(K) \rightarrow K$ is continuous.

The remaining proof follows a similar way as done in Theorem 2.2 of [9].

Remark 2.1 Under the assumptions of Theorem 2.1, we are unable to assert that $(I-T)^{-1}$ : $(I-T)(M) \rightarrow E$ is continuous. However, this is unimportant for our purpose.

Let us now investigate the case in which we can first establish a nonempty, compact convex, possible fixed point subset of the set $\mathcal{F}(E, K ; T, S)$. In such case, the condition (iii) above is not needed as illustrated in the following corrected version of Theorem 1.2.

Theorem 2.2 Let $K \subset E$ be a nonempty, bounded, closed and convex subset, $K \subset M \subset E$ and $T: M \rightarrow E$ be a map. Suppose that $S: K \rightarrow E$ is continuous such that

(i) $S(K) \subset(I-T)(M)$ and $[x=T x+S y, y \in K] \Longrightarrow x \in K($ or $S(K) \subset(I-T)(K))$;

(ii) $\psi(T(A)+S(A))<\psi(A)$ for all $A \subset K$ with $\psi(A)>0$;

(iii) $T$ is closed in $\mathcal{F}$; that is, if $\left\{x_{n}\right\} \subset \mathcal{F}$ for which $x_{n} \rightarrow x$ and $T x_{n} \rightarrow y$, then $y=T x$;

(iv) for each $z$ in the range of $S$, the set $\Delta_{z}=\{x \in M:(I-T) x=z\}$ is convex.

Then there exists a point $x^{*} \in K$ with $S x^{*}+T x^{*}=x^{*}$.

Proof We only prove the case when $I-T$ is injective. The otherwise case can be shown similarly as in [9].

By means of the technique of measures of noncompactness, the essentially same argument as in Theorem 2.2 of [9] shows that there exists a nonempty, compact and convex set $A_{0}$ of $K$ such that $(I-T)^{-1} S: A_{0} \rightarrow A_{0}$. We next show in the absence of the condition (iii) in Theorem 1.2 that $(I-T)^{-1} S: A_{0} \rightarrow A_{0}$ is still continuous. To achieve this, let $x_{n}, x \in A_{0}$ with $x_{n} \rightarrow x$, and put $y_{n}=(I-T)^{-1} S x_{n}$ and $y=(I-T)^{-1} S x$. Then $y_{n}, y \in A_{0} \cap \mathcal{F}$. Furthermore, one can readily deduce that $y_{n_{k}} \rightarrow y_{0}$ for some $y_{0}$ and $T y_{n_{k}} \rightarrow y_{0}-S x$. By employing the closedness of $T$ in $\mathcal{F}$, one concludes that $y_{0}-S x=T y_{0}$, i.e., $y_{0}=(I-T)^{-1} S x=y$. Consequently,

$$
(I-T)^{-1} S x_{n_{k}} \rightarrow(I-T)^{-1} S x .
$$

Now an analogous argument to that of Theorem 2.1 yields that

$$
(I-T)^{-1} S x_{n} \rightarrow(I-T)^{-1} S x .
$$

This verifies the continuity of $(I-T)^{-1} S$ on the set $A_{0}$.

Remark 2.2 The resulting mapping $(I-T)^{-1} S: K \rightarrow K$ may not be continuous. 
If the compactness hypothesis is imposed on the set $\mathcal{F}(M, K ; T, S)$, then the item (iii) in Theorem 1.2 is redundant. And the boundedness of $K$ is also not required. The following is a refined version of Theorem 2.3 in [9].

Theorem 2.3 Let $K \subset E$ be a nonempty, closed and convex subset, $K \subset M \subset E$ and $T$ : $M \rightarrow E$ be a map. Suppose that $S: K \rightarrow E$ is continuous such that

(i) $S(K) \subset(I-T)(M)$ and $[x=T x+S y, y \in K] \Longrightarrow x \in K($ or $S(K) \subset(I-T)(K))$;

(ii) the set $\mathcal{F}(M, K ; T, S)$ is relatively compact;

(iii) if $\left\{x_{n}\right\} \subset \mathcal{F}$ for which $x_{n} \rightarrow x$ and $T x_{n} \rightarrow y$, then $y=T x$;

(iv) for each $z \in S(K)$, the set $\Delta_{z}=\{x \in M:(I-T) x=z\}$ is convex.

Then there exists a point $x^{*} \in K$ such that $S x^{*}+T x^{*}=x^{*}$.

Proof It is enough to demonstrate, when $I-T$ is one-to-one, that the resulting mapping $N:=(I-T)^{-1} S: K \rightarrow K$ is compact and continuous. In fact, let $y_{n} \in N(K)$, then $(I-T) y_{n}=$ $S x_{n}$ for some $x_{n} \in K$ and hence $y_{n} \in \mathcal{F}$ by the definition of $\mathcal{F}$. Keeping assumption (ii) in mind, one obtains that $\left\{y_{n}\right\}$ possesses a convergent subsequence. Let now $\left\{x_{n}\right\} \subset K$ with $x_{n} \rightarrow x$ in $K$. Put $y_{n}=N x_{n}$ and $y=N x$. Then $y_{n} \in \mathcal{F}$ and $(I-T) y_{n} \rightarrow S x$ by the continuity of $S$. One knows from (iii) that there exists a subsequence $\left\{y_{n_{k}}\right\}$ of $\left\{y_{n}\right\}$ such that $y_{n_{k}} \rightarrow y_{0}$ for some $y_{0} \in K$. Consequently, one infers as before that $y_{0}=N x=y$ and $y_{n} \rightarrow y$. This confirms the assertion.

Points made in $\mathrm{p}_{3}$ and $\mathrm{p}_{5}$ in the Introduction are summarized in the following proposition.

Proposition 2.1 Let the conditions (i) and (iii) of Theorem 2.1 hold. Then the precompactness of $S(K)$ implies the precompactness of $\mathcal{F}(M, K ; T, S)$, where $M$ is a closed subset of $E$. Conversely, if $T$ is continuous on $\mathcal{F}(M, K ; T, S)$, then the precompactness of $\mathcal{F}(M, K ; T, S)$ implies the precompactness of $S(K)$.

Proof For any $x_{n} \in \mathcal{F}$, there is $y_{n} \in K$ so that $x_{n}=T x_{n}+S y_{n}$. The relative compactness of $S(K)$ tells us there exists a subsequence $\left\{x_{n_{k}}\right\}$ of $\left\{x_{n}\right\}$ for which $(I-T) x_{n_{k}} \rightarrow$ some $z$. The assumption (iii) now implies that $\left\{x_{n}\right\}$ possesses a convergent subsequence. This means that $\mathcal{F}(M, K ; T, S)$ is precompact. For the second part, let $y_{n} \in K$, we have to show that $\left\{S y_{n}\right\}$ has a convergent subsequence. Taking the item (i) into account, there exists $x_{n} \in M$ satisfying $(I-T) x_{n}=S y_{n}$. In light of the fact that $x_{n} \in \mathcal{F}$ and $\mathcal{F}$ is precompact, it follows that there exists a subsequence $\left\{x_{n_{k}}\right\}$ of $\left\{x_{n}\right\}$ so that $x_{n_{k}} \rightarrow x$ for some $x \in M$. The continuity of $T$ on $\mathcal{F}$ immediately yields $S y_{n_{k}} \rightarrow x-T x$, which establishes the relative compactness of $S(K)$.

Typically, the condition $S(K) \subset(I-T)(M)$ is verified by showing that $I-T$ is surjective. Surjectivity result of this type has been studied widely, for instance, see [14-16]. The above refined formulations of the Krasnosel'skii fixed point theorems allow us to derive new classes of Krasnosel'skii type theorems. Here, as an illustration, we apply Corollary 2 of [16] together with Theorem 2.3 to derive one paradigm of that new type of Krasnosel'skii fixed point theorem. As usual, we say a continuous map $T: D(T) \subset E \rightarrow E$ is condensing if $\psi(T(A))<\psi(A)$ for all bounded $A \subset D(T)$ with $\psi(A)>0$.

Theorem 2.4 Let $K \subset E$ be a nonempty, closed and convex subset. Suppose that $S: K \rightarrow E$ and $T: E \rightarrow E$ are two continuous maps which satisfy the following conditions: 
(i) $S(K) \subset(I-T)(E)$ and $[x=T x+S y, y \in K] \Longrightarrow x \in K($ or $S(K) \subset(I-T)(K))$;

(ii) the set $S(K)$ is relatively compact;

(iii) $T$ is condensing and $\lim \sup _{\|x\| \rightarrow \infty}\|T x\| /\|x\|<1$;

(iv) for each $z \in S(K)$, the set $\Delta_{z}=\{x \in E:(I-T) x=z\}$ is convex.

Then there exists a point $x^{*} \in K$ such that $S x^{*}+T x^{*}=x^{*}$.

Proof (a) We shall show that $I-T$ is surjective and so $S(K) \subset(I-T)(E)$. Indeed, since $T$ is condensing, it is well known that $(I-T)$ maps closed sets into closed sets. Then, with assumption (iii), Corollary 2 of [16] gives that $I-T$ is surjective.

(b) To prove, if $\left\{x_{n}\right\} \subset \mathcal{F}(E, K ; T, S)$ and $(I-T) x_{n} \rightarrow y$, then $\left\{x_{n}\right\}$ possesses a convergent subsequence. As a matter of fact, setting $y_{n}=(I-T) x_{n}$, from the relation

$$
\psi\left(\left\{x_{n}\right\}\right) \leq \psi\left(\left\{y_{n}\right\}\right)+\psi\left(\left\{T x_{n}\right\}\right)=\psi\left(\left\{T x_{n}\right\}\right)
$$

and the fact that $T$ is condensing, we conclude $\psi\left(\left\{x_{n}\right\}\right)=0$, implying that $\left\{x_{n}\right\}$ has a convergent subsequence.

(c) The set $\mathcal{F}$ is relatively compact. This follows from (b), (ii) and Proposition 2.1.

(d) If $\left\{x_{n}\right\} \subset \mathcal{F}$ for which $x_{n} \rightarrow x$ and $T x_{n} \rightarrow y$, then $y=T x$ by the continuity of $T$.

Now, an easy application of Theorem 2.3 ensures the existence of $x^{*} \in K$ such that $S x^{*}+$ $T x^{*}=x^{*}$.

Remark 2.3 If $T$ is a contraction then (iii) is true. The second requirement in (iii) seems new in the context of Krasnosel'skii type fixed point theorem. We note that the surjectivity of $I-T$ may be proved via index theory for condensing operators [14]. So the second part of (iii) may be replaced by $\liminf _{\|x\| \rightarrow \infty}\|T x\| /\|x\|>1$ without affecting the conclusion of the theorem.

When $T: E \rightarrow E$ is nonexpansive, i.e., $\|T x-T y\| \leq\|x-y\|$ for all $x, y \in E$, we can also use Theorem 2.3 to obtain two fixed point results of this type.

Theorem 2.5 Let $K \subset$ E be a nonempty, bounded, closed and convex subset. Suppose that $S: K \rightarrow E$ and $T: E \rightarrow E$ satisfy the following conditions:

(i) $[\lambda \in(0,1), x=\lambda T x+\lambda S y, y \in K] \Longrightarrow x \in K$;

(ii) $S$ is continuous and the set $S(K)$ is relatively compact;

(iii) if $(I-T) x_{n} \rightarrow y$, then $\left\{x_{n}\right\}$ possesses a convergent subsequence $\left\{x_{n_{k}}\right\}$;

(iv) $T$ is nonexpansive on $E$.

Then there exists a point $x^{*} \in K$ such that $S x^{*}+T x^{*}=x^{*}$.

Proof For each $n \geq 2$, let $\lambda_{n}=1-1 / n$. Since $\lambda_{n} T: E \rightarrow E$ is a contraction, we have $I-\lambda_{n} T$ is surjective onto $E$. Then it is straightforward to check that all the conditions of Theorem 2.3 are satisfied for $\lambda_{n} T, \lambda_{n} S$ and $M=E$. Hence, by (i) there exists a sequence $x_{n} \in K$ such that

$$
\frac{1}{n-1} x_{n}=-(I-T) x_{n}+S x_{n}
$$

Recall that $K$ is bounded and $S(K)$ is precompact, passing eventually to a subsequence as $n \rightarrow \infty$ in (2.1), we get $(I-T) x_{n} \rightarrow y$ for some $y$. So, up to a further subsequence, we have by (iii) $x_{n} \rightarrow x^{*}$. Then passing to the limit in (2.1) again, we find $x^{*}=T x^{*}+S x^{*}$. 
Remark 2.4 The condition (i) is motivated by the condition $(\mathscr{L})$ in Barroso [4].

In Theorem 2.5, if $K$ happens to be a compact set, then (iii) is not needed and (i) can have two alternatives. Precisely, we have the following result, its proof is similar to Theorem 2.5 and is an application of Theorem 2.3.

Theorem 2.6 Let $K \subset E$ be a nonempty, compact and convex subset. Let $S: K \rightarrow E$ be continuous and $T: E \rightarrow E$ be nonexpansive. Suppose

(i) either $[\lambda \in(0,1), x=\lambda T x+\lambda S y, y \in K] \Longrightarrow x \in K$ or

(ii) $[\lambda \in(0,1), x=\lambda T x+S y, y \in K] \Longrightarrow x \in K$.

Then there exists a point $x^{*} \in K$ such that $S x^{*}+T x^{*}=x^{*}$.

Remark 2.5 We observed that there exist several other papers studying fixed point theorems for $T+S$ with $T$ being nonexpansive, see, for instance, Edmunds [13] and Agarwal, O'Regan and Taoudi [7]. Comparing their results with Theorems 2.5 and 2.6, we present other alternative conditions instead of requiring $S$ to be weakly-strongly continuous, $T$ to be sort of 'weak sequential continuity' and $T(K)+S(K) \subset K$. In some sense, our results extend and complement theirs.

In the end of this note, we provide three simple examples to show that the continuity of the composite mapping $T \circ S$ on some set does not guarantee the continuity of its components even for the case that both $T$ and $S$ are linear. To see this, let $T: D(T) \subset X \rightarrow X$ and $S: D(S) \subset E \rightarrow X$ be two mappings, where $X$ is a linear normed or metric space, $D(T)$ and $D(S)$ are the domains of $T$ and $S$, respectively. Assume that $S(D(S)) \subset D(T)$. These examples are probably known in functional analysis courses; however, we shall include them here for convenience, and there proofs are easy and hence are omitted.

Example 2.1 Let $c_{00}=\left\{x(n) \in c \mid\right.$ there exists $n_{0} \geq 1$ such that $x(n)=0$ for all $\left.n \geq n_{0}\right\}$. Define $T: c_{00} \rightarrow c_{00}$ by

$$
(T x)(n)=\frac{1}{n} x(n), \quad n=1,2, \ldots
$$

Then $T$ is bijective, linear and bounded, but $T^{-1}: c_{00} \rightarrow c_{00}$ is unbounded.

Example 2.2 Let $\left\{a_{k}\right\} \subset \mathbb{R}$ be a sequence satisfying $\sup _{k \geq 1}\left|a_{k}\right|<\infty$. Define $T: l^{1} \rightarrow l^{1}$ by

$$
T x=\left\{a_{k} \xi_{k}\right\}, \quad \forall x=\left\{\xi_{k}\right\} \in l^{1} .
$$

Then $T$ has a bounded inverse if and only if $\inf _{k \geq 1}\left|a_{k}\right|>0$.

Remark 2.6 In view of Example 2.2 and the Banach inverse mapping theorem, we know that the operator $T: l^{1} \rightarrow l^{1}$ defined by

$$
T x=\left\{\frac{1}{k} \xi_{k}\right\}, \quad \forall x=\left\{\xi_{k}\right\} \in l^{1}
$$

is not a surjective map onto $l^{1}$. 
Example 2.3 Let $X=\left\{\left(\xi_{1}, \xi_{2}, \ldots, \xi_{n}, \ldots\right)\left|\sum_{n=1}^{\infty}\right| \xi_{n} \mid<\infty\right\}$ be a linear normed space with the norm $\|x\|=\sup _{n \geq 1}\left|\xi_{n}\right|$. For $a=(1,-1,0, \ldots) \in X$, we defined $T: X \rightarrow X$ by

$$
T x=x-a f(x), \quad \forall x=\left(\xi_{1}, \xi_{2}, \ldots\right) \in X
$$

where $f: X \rightarrow \mathbb{R}$ is given by

$$
f(x)=\sum_{n=1}^{\infty} \xi_{n}, \quad \forall x=\left(\xi_{1}, \xi_{2}, \ldots\right) \in X
$$

Then $T$ is not continuous, $N(T)=\theta$ (the zero element) and $T^{-1}: X \rightarrow X$ is not continuous.

\section{Competing interests}

The authors declare that they have no competing interests.

\section{Authors' contributions}

All authors contributed equally to the writing of this paper. All authors read and approved the final manuscript.

\section{Author details}

${ }^{1}$ Institute for Mathematical Sciences, Renmin University of China, Beijing, 100872, China. ${ }^{2}$ School of Mathematical Sciences, Beijing Normal University, Beijing, 100875, China.

\section{Acknowledgements}

Part of the work was done when TX was visiting the School of Mathematical Sciences, Beijing Normal University; he wishes to thank Prof. Rong Yuan for kind invitation and the school for hospitality. The authors thank the referees very much for their helpful comments and suggestions, which further improved the exposition of this work. TX was supported by the Fundamental Research Funds for the Central Universities and the Research Funds of Renmin University of China (No. 15XNLF10), and China Postdoctoral Science Foundation (No. 2015M570190). RY was supported by NSF of China and RFDP.

Received: 11 March 2015 Accepted: 14 June 2015 Published online: 01 July 2015

\section{References}

1. Krasnosel'skii, MA: Two remarks on the method of successive approximations. Usp. Mat. Nauk 10, 123-127 (1955) (in Russian)

2. Krasnosel'skii, MA: Some problems of nonlinear analysis. Transl. Am. Math. Soc. 10(2), 345-409 (1958)

3. Burton, T: A fixed-point theorem of Krasnosel'skii. Appl. Math. Lett. 11(1), 85-88 (1998)

4. Barroso, CS: Krasnosel'skii's fixed point theorem for weakly continuous maps. Nonlinear Anal. 55(1-2), 25-31 (2003)

5. Park, S: Generalizations of the Krasnosel'skii fixed point theorem. Nonlinear Anal. 67(12), 3401-3410 (2007)

6. Liu, YC, Li, ZX: Krasnosel'skii type fixed point theorems and applications. Proc. Am. Math. Soc. 136, 1213-1220 (2008)

7. Agarwal, RP, O'Regan, D, Taoudi, MA: Browder-Krasnosel'skii-type fixed point theorems in Banach spaces. Fixed Point Theory Appl. 2010, 243716 (2010)

8. Garcia-Falset, J: Existence of fixed points for the sum of two operators. Math. Nachr. 283, 1736-1757 (2010)

9. Xiang, T, Yuan, R: Critical type of Krasnosel'skii fixed point theorem. Proc. Am. Math. Soc. 139, 1033-1044 (2011)

10. Burton, TA, Zhang, B: Fractional equations and generalizations of Schaefer's and Krasnosel'skii's fixed point theorems. Nonlinear Anal. TMA 75, 6485-6495 (2012)

11. Burton, TA, Purnaras, IK: A unification theory of Krasnosel'skii for differential equations. Nonlinear Anal. 89, 121-133 (2013)

12. Berzig, M, Chandok, S, Khan, M: Generalized Krasnosel'skii fixed point theorem involving auxiliary functions in bimetric spaces and application to two-point boundary value problem. Appl. Math. Comput. 248, $323-327$ (2014)

13. Edmunds, DE: Remarks on non-linear functional equations. Math. Ann. 174, 233-239 (1967)

14. Nussbaum, RD: The fixed point index for local condensing maps. Ann. Mat. Pura Appl. (4) 89, 217-258 (1971)

15. Nussbaum, RD: Degree theory for local condensing maps. J. Math. Anal. Appl. 37, 741-766 (1972)

16. Petryshyn, WV: Remarks on condensing and k-set-contractive mappings. J. Math. Anal. Appl. 39, 717-741 (1972) 